\title{
Uso de la función semivariograma y estimación kriging en el análisis espacial de un indicador entomológico de Aedes aegypti (Diptera: Culicidae)
}

\begin{abstract}
Larry Niño
Laboratorio de Entomología, Secretaría Seccional de Salud del Meta, Villavicencio, Colombia

Introducción. Aedes aegypti es el principal vector del dengue en América. La prevención y control de esta enfermedad requieren de nuevas técnicas de vigilancia para este mosquito. El análisis de la distribución espacial de estas poblaciones puede llegar a jugar un papel importante en la planificación y evaluación de medidas orientadas a la disminución del contacto vector-hombre.

Objetivo. Analizar y representar gráficamente el patrón de variación espacial del indicador aédico correspondiente al índice de recipientes, definido como el porcentaje de depósitos con agua infestados con larvas de $A$. aegypti.

Materiales y métodos. Se realizaron encuestas sobre larvas en la totalidad de las viviendas del barrio La Independencia (Acacías, Meta) en mayo de 2007, con las cuales se calcularon los índices de recipientes en cada manzana. La metodología empleada en el análisis de este indicador correspondió a la estadística espacial, concretamente a la función semivariograma junto con estimaciones kriging.

Resultados. El semivariograma experimental obtenido se ajustó al modelo matemático de Gauss, cuya meseta se calculó en 5,1, el rango en 57,1 m y la pepita en 0,09. Se construyó una gráfica bidimensional de la estimación kriging que permitió identificar las manzanas con mayores índices de recipientes.

Conclusión. El análisis y la representación gráfica de la distribución del índice de recipientes pueden ser útiles en la vigilancia, la toma y la evaluación de acciones contra la infestación de A. aegypti.
\end{abstract}

Palabras clave: Aedes aegypti, modelos estadísticos, vigilancia epidemiológica, vectores de enfermedades, mapa de riesgo, Colombia.

\section{Use of the function semivariogram and kriging estimation in the spacial analysis of Aedes aegypti (Diptera: Culicidae) distributions}

Introduction. Aedes aegypti is the main vector of dengue in the Americas. The prevention and control of this disease require new monitoring techniques for this mosquito. Knowledge of the spatial and temporal distributions of $A$. aegypti populations allow the planning and evaluation of measures to decrease the vector-human contact.

Objective. The spatial variation pattern of the $A$. aegypti container index (defined as the percentage of artificial containers infested with $A$. aegypti larva) was analyzed for the purpose of developing a graphical representation.

Materials and methods. Larval surveys were undertaken in every household of La Independencia neighborhood in May 2007 (Acacías-Meta). Spatial statistics employing the semivariogram function and kriging estimations were applied to these data.

Results. The experimental semivariogram output was adjusted to the gaussian mathematical model, whose sill was calculated to be 5.1 , the range as 57.1 meters and the nugget as 0.01 . A bidimensional graph of the kriging estimation was built, allowing the identification of the urban areas with highest container index.

Conclusion. The analysis of the container index and distribution map provided a useful tool in monitoring, evaluating and making control decisions concerning $A$. aegypti infestations. 
Key words: Aedes aegypti; models, statistical; epidemiological surveillance, disease vectors, risk map, Colombia.

Los brotes por dengue, en su manifestación clásica y hemorrágica, acarrean grandes gastos al sector salud por hospitalización, asistencia médica y planes de emergencia con fines de erradicación del vector (1). Aedes aegypti se considera como el principal vector de esta enfermedad en las Américas; en Colombia se encuentra comúnmente por debajo de los $1.800 \mathrm{~m}$, aunque se ha reportado en municipios con más de 2.000 metros de altura (2). En la actualidad, las medidas de control y la prevención del dengue se basan en tratamientos químicos, físicos, biológicos y educativos contra el vector, pero la eficacia de estas acciones depende de una adecuada vigilancia entomológica. Las variaciones de los indicadores entomológicos en el tiempo y el espacio permiten planificar estrategias encaminadas a disminuir el contacto vector-hombre (3).

En el pasado, el análisis de las distribuciones espaciales se realizaba comúnmente mediante índices de dispersión, ignorando la localización de las muestras e imposibilitando su distinción en el espacio (4). En la actualidad, la tecnología de los sistemas de información geográfica y la geoestadística son aplicadas con éxito en el estudio de insectos al relacionar estos organismos con características del medio donde se desarrollan $(5,6)$.

Un sistema de información geográfica es un conjunto de programas informáticos que captan, almacenan, recuperan, transforman, muestran y analizan diversos tipos de datos geográficamente referenciados. Esta información busca generar mapas temáticos o coberturas que pueden combinarse con el fin de analizar las posibles interacciones entre las diversas variables (7).

Aunque los sistemas de información geográfica han permitido a los investigadores la manipulación

\footnotetext{
Correspondencia:

Larry Niño, Laboratorio de Entomología, Secretaría Seccional de Salud del Meta, Carrera 37 № 41-80, Barrio Barzal Alto, Villavicencio, Meta.

Teléfax: (578) 6620129

Ininoa@unal.edu.co

Recibido: 30/04/08; aceptado:20/08/08
}

de datos espaciales, la caracterización y la creación de modelos de los patrones de distribución espacial, son muy poco factibles si no se cuenta con la herramienta estadística fundamental para este fin: la geoestadística $(6,8)$.

La geoestadística es una rama de la estadística aplicada que comprende un conjunto de herramientas y técnicas que sirven para analizar y predecir los valores de una variable que se muestra distribuida en el espacio de una forma continua; se basa en la teoría de variables regionalizadas y sus diferencias de autocorrelación, en la que la única suposición requerida es que la varianza entre muestras sea función de las distancias de separación. Las tendencias superficiales y los métodos de interpolación proceden de la geoestadística, pero sus consideraciones, implícitas o explícitas, van dirigidas a la creación de mapas de superficie basados en las observaciones obtenidas. La autocorrelación espacial hace referencia a la correlación de la variable en diferentes direcciones y distancias de separación, examinándose por medio de la estimación de semivariogramas junto con su posterior modelado al incorporarse a un procedimiento de interpolación superficial denominado kriging (9).

La idea básica de kriging, al igual que la geoestadística, corresponde a la noción de dependencia espacial en la cual las muestras cercanas tienen mayor similitud entre sí que las dispuestas apartadamente. Con esta información, el kriging estima las variables en los puntos no muestrales basándose en las muestras conocidas (6).

\section{Materiales y métodos}

El área de estudio se localiza en el municipio de Acacías perteneciente al departamento del Meta; este municipio cuenta con una extensión de 1.169 $\mathrm{km}^{2}$ a una altura sobre el nivel del mar de $498 \mathrm{~m}$ y una temperatura media de $24^{\circ} \mathrm{C}$. Actualmente, Acacías carece de continuidad en el suministro de agua y, debido al arraigo cultural, el vaciamiento de residuos sólidos en el peridomicilio es una práctica común en algunos sectores del poblado, 
lo cual contribuye a la formación de criaderos de $A$. aegypti dentro y fuera de las viviendas.

Los datos se obtuvieron de las encuestas sobre larvas de $A$. aegypti realizadas en la totalidad de las viviendas del barrio Independencia en mayo de 2007, por personal calificado y capacitado para este objetivo. En primera instancia, cada una de las 56 manzanas del barrio en cuestión fue numerada y referenciada geográficamente para lograr la regionalización de la variable índice de recipientes, definida como el porcentaje de depósitos con agua infestados con larvas de $A$. aegypti (cuadro 1, figura 1), la cual se calculó de la siguiente manera:

índice de recipientes $=\mathrm{N}^{\circ}$ recipientes infestados $/$ $N^{\circ}$ recipientes revisados $\times 100$.

El análisis espacial se llevó a cabo en tres etapas (8). En la primera, se realizó un análisis exploratorio, en el que se examinaron los datos sin tener en cuenta su distribución geográfica, se eliminaron los erróneos y se identificó la distribución que seguían con el cálculo de algunos parámetros de estadística descriptiva.

En la segunda etapa se estudió la continuidad o dependencia espacial de la variable con el cálculo del semivariograma isotrópico experimental y su posterior ajuste a uno teórico, lo cual permitió cuantificar el grado y la escala de la variación espacial. El semivariograma se calculó con la ecuación (9):

$$
\gamma_{(\mathrm{h})}=1 / 2 \mathrm{~N}_{(\mathrm{h})} \Sigma\left[\mathrm{Z}_{(\mathrm{x})}-\mathrm{Z}_{(\mathrm{x}+\mathrm{h})}\right]^{2}
$$

donde, $\gamma_{(h)}$ es la semivarianza del índice de recipientes para todas las manzanas localizadas en el espacio separado por los intervalos de las distancias $\mathrm{h} ; \mathrm{N}_{(\mathrm{h})}$ corresponde al número total de pares de manzanas separadas por un intervalo de distancia $h ; Z_{(x)}$ es el valor del índice en una localización $x ; y Z_{(x+h)}$ es el valor del índice a la distancia del intervalo $\mathrm{h}$ desde $\mathrm{x}$.

Una vez se estimaron los semivariogramas experimentales, se hizo necesario ajustarlos a modelos teóricos, ya que los experimentales carecen de una función matemática precisa, lo que los hace imposible de trabajar. Estos modelos se dividen en acotados, si el semivariograma tiende asintóticamente a la semivarianza, correspondientes al esférico, exponencial y de Gauss; y en no acotados, si el semivariograma tiende al infinito cuando la distancia así lo haga, que incluyen al modelo lineal, el logarítmico y el potencial $(8,9)$.

Como el semivariograma representa la tasa media de cambio de una propiedad con la distancia, los modelos acotados aseguran que la covarianza de los incrementos sea finita. Estos modelos son ampliamente usados cuando se evidencia un buen ajuste y presentan los siguientes parámetros (6,9-11).

Meseta: debido a que el semivariograma es una función monótona creciente, ésta alcanza un valor límite denominado meseta y es equivalente a la varianza de la población. Se define también como la cota superior del semivariograma o su límite superior cuando la distancia (h) tiende al infinito.

Rango: corresponde a la distancia (h) donde se alcanza la meseta. Determina la zona de influencia en torno a un punto o distancia promedio a la cual las muestras se correlacionan espacialmente, más allá de la cual la autocorrelación es nula. Si el semivariograma no tiende asintóticamente hacia la semivarianza, es decir que no existe una meseta, significa que el rango es superior a la distancia máxima representada.

Pepita: es una discontinuidad puntual del semivariograma en el origen. Puede deberse a que la variabilidad espacial tiene una escala más pequeña que la mínima distancia considerada o a un error experimental.

Finalmente, se realizó una estimación kriging, la cual se basa en la idea fundamental de la dependencia espacial: los lugares que disten menos entre sí tendrán unos valores de los atributos más semejantes que los correspondientes a los puntos o bloques que estén más separados. Todos los estimadores del tipo kriging no son sino variantes del estimador lineal básico $Z^{*}{ }_{(x)}$, definido como (9):

$$
Z_{(x)}^{*}-m_{(x)}=\Sigma \omega_{(\mathrm{i})}\left[Z_{(x)}-m_{(x i)}\right]
$$

donde, $\omega_{i)}$ son los pesos asignados a los datos $\mathrm{z}_{(\mathrm{xi})}$, los cuales se relacionan con la magnitud $\mathrm{y}$ proximidad de las muestras y cuyos atributos son 
Cuadro 1. Coordenadas e índice de recipientes de las manzanas del barrio La Independencia (municipio de Acacías).

\begin{tabular}{|c|c|c|c|c|c|}
\hline Manzana & $\mathbf{X}$ & $\mathbf{Y}$ & $\begin{array}{l}\text { Total de } \\
\text { recipientes }\end{array}$ & $\begin{array}{l}\text { Recipientes } \\
\text { positivos }\end{array}$ & $\begin{array}{l}\text { Indíce de } \\
\text { recipientes }\end{array}$ \\
\hline 2 & 1291191.8163 & 279235.7453 & 136 & 6 & 4,41 \\
\hline 3 & 1291284.2498 & 279312.2510 & 102 & 8 & 7,84 \\
\hline 5 & 1291242.4012 & 279229.9212 & 54 & 1 & 1,85 \\
\hline 6 & 1291308.2333 & 279283.7126 & 104 & 8 & 7,69 \\
\hline 7 & 1291401.9565 & 279358.1980 & 420 & 9 & 2,14 \\
\hline 8 & 1291326.1373 & 279253.0857 & 92 & 1 & 1,09 \\
\hline 9 & 1291458.3445 & 279363.6487 & 211 & 10 & 4,74 \\
\hline 11 & 1291380.6637 & 279215.2490 & 55 & 2 & 3,64 \\
\hline 12 & 1291459.8862 & 279313.1800 & 305 & 4 & 1,31 \\
\hline 13 & 1291422.6093 & 279162.0856 & 46 & 1 & 2,17 \\
\hline 14 & 1291442.0841 & 279188.4653 & 196 & 10 & 5,10 \\
\hline 15 & 1291462.6836 & 279217.8206 & 134 & 3 & 2,24 \\
\hline 16 & 1291486.8076 & 279249.9848 & 65 & 0 & 0,00 \\
\hline 17 & 1291502.1857 & 279312.8001 & 66 & 1 & 1,52 \\
\hline 18 & 1291522.7853 & 279281.6044 & 39 & 0 & 0,00 \\
\hline 19 & 1291495.6457 & 279151.8189 & 152 & 7 & 4,61 \\
\hline 21 & 1291477.8968 & 279105.9422 & 217 & 5 & 2,30 \\
\hline 22 & 1291567.8046 & 279141.8090 & 36 & 2 & 5,56 \\
\hline 23 & 1291497.1922 & 279069.9985 & 84 & 2 & 2,38 \\
\hline 24 & 1291493.3283 & 279034.8279 & 174 & 9 & 5,17 \\
\hline 25 & 1291484.8296 & 279000.5686 & 123 & 5 & 4,07 \\
\hline 26 & 1291486.4780 & 278961.5306 & 153 & 8 & 5,23 \\
\hline 29 & 1291594.8084 & 278881.8493 & 168 & 6 & 3,57 \\
\hline 30 & 1291644.3025 & 278893.7939 & 40 & 0 & 0,00 \\
\hline 31 & 1291643.3668 & 278848.0007 & 50 & 2 & 4,00 \\
\hline 32 & 1291485.2175 & 278819.0626 & 111 & 7 & 6,31 \\
\hline 33 & 1291485.6780 & 278779.6842 & 143 & 6 & 4,20 \\
\hline 34 & 1291481.7414 & 278746.9007 & 141 & 7 & 4,97 \\
\hline 36 & 1291586.0479 & 278777.5298 & 285 & 7 & 2,46 \\
\hline 38 & 1291659.4576 & 278809.6940 & 5 & 0 & 0,00 \\
\hline 41 & 1291428.3106 & 278679.6098 & 141 & 9 & 6,38 \\
\hline 42 & 1291467.0711 & 278676.1773 & 173 & 12 & 6,94 \\
\hline 43 & 1291502.6124 & 278674.4599 & 103 & 6 & 5,83 \\
\hline 44 & 1291579.9296 & 278702.9303 & 123 & 2 & 1,63 \\
\hline 45 & 1291580.4629 & 278666.7758 & 378 & 14 & 3,70 \\
\hline 46 & 1291575.6439 & 278627.0240 & 108 & 9 & 8,33 \\
\hline 47 & 1291649.2378 & 278675.0419 & 19 & 1 & 5,26 \\
\hline 48 & 1291391.1161 & 278572.5408 & 50 & 3 & 6,00 \\
\hline 49 & 1291461.2437 & 278600.3413 & 136 & 6 & 4,41 \\
\hline 50 & 1291421.9403 & 278554.0716 & 36 & 0 & 0,00 \\
\hline 51 & 1291460.0753 & 278549.7804 & 108 & 9 & 8,33 \\
\hline 52 & 1291496.8238 & 278553.1602 & 15 & 1 & 6,67 \\
\hline 53 & 1291537.7222 & 278572.6792 & 157 & 4 & 2,55 \\
\hline 54 & 1291573.0211 & 278569.9516 & 189 & 3 & 1,59 \\
\hline 55 & 1291608.7078 & 278568.7833 & 81 & 0 & 0,00 \\
\hline 56 & 1291640.0798 & 278567.2175 & 36 & 1 & 2,78 \\
\hline
\end{tabular}




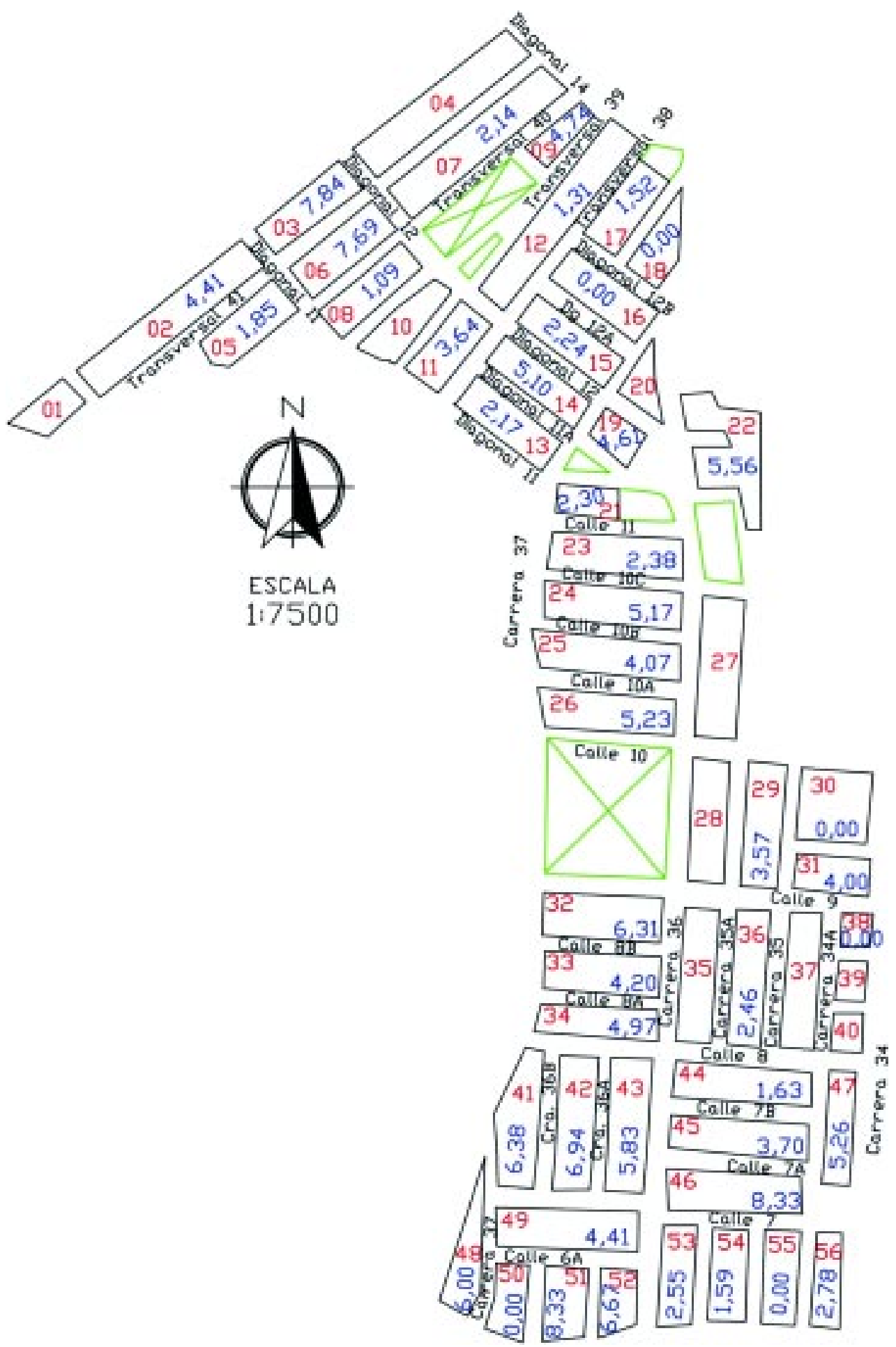

Figura 1. Barrio La Independencia (municipio de Acacías). Numeración de manzanas e índice de recipientes, en rojo y azul, respectivamente. 
estimados en el semivariograma. Los valores esperados de las variables aleatorias $Z_{(x)}$ y $Z_{(x)}$ son $m_{(x)}$ y $m_{(x i)}$, respectivamente. El número de datos, $n$, considerado en la estimación, varía de un lugar a otro. En la práctica se emplean los datos existentes en las proximidades del punto por estimar, dentro de un entorno definido al principio.

\section{Resultados}

Durante la primera fase del análisis fue corroborada la normalidad de los datos con el cálculo de los estadísticos descriptivos (cuadro 2) y la prueba de Kolmogorov-Smirnov, la cual arrojó un estadístico de 0,099 con una significancia de 0,2.

En la segunda parte del estudio, el semivariograma experimental fue ajustado al modelo matemático teórico correspondiente al de Gauss por el programa GS+ Geostatistics for the Environmental Sciences, version 7.0 (versión de prueba) de forma automática (figura 2), ya que no se cuenta con información a priorique permita un ajuste más riguroso. Este modelo se caracteriza por alcanzar la meseta asintóticamente y define el rango como la distancia a la cual el valor del semivariograma alcanza el $95 \%$ del valor de la meseta. El modelo de Gauss se define como:

$\gamma_{(\mathrm{h})}=1-\exp \left(-3 \mathrm{~h}^{2} / \mathrm{a}^{2}\right)$

donde $\gamma_{(h)}$ es la semivarianza en el intervalo de distancia $h$, y a es el rango.

En los parámetros estructurales calculados para el semivariograma se observó que la meseta calculada fue de 5,1, la cual, es una medida de la variación espacial y, además, equivale a la varianza de la población. La proximidad de estos valores, correspondientes a la meseta y la varianza de la

Cuadro 2. Valores estadísticos descriptivos calculados para la variable índice de recipientes.

\begin{tabular}{lr}
\hline Estadístico & Valor \\
\hline Media & 3,72 \\
Mediana & 3,85 \\
Varianza & 5,83 \\
Desviación típica & 2,42 \\
Asimetría & 0,12 \\
Curtosis & $-0,87$ \\
\hline
\end{tabular}

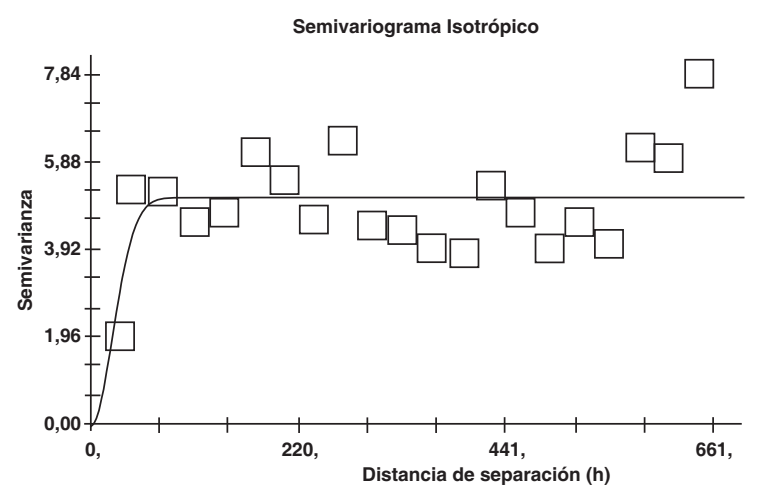

Figura 2. Semivariograma isotrópico calculado para el índice de recipientes

población (cuadro 2), es síntoma de un buen ajuste del semivariograma.

El rango presentó un valor de 57,15, el cual corresponde a la distancia en metros en la que se alcanza la meseta y donde se determina la zona de influencia en torno a un punto y en la cual se correlaciona el índice; más allá de esta distancia se considera que la relación es nula.

La pepita es la intercepción con el eje Y; se calculó con un valor de 0,01 y se considera como una discontinuidad puntual del semivariograma en el origen (se espera que a distancia $=0$, varianza $=0$ ). Cuando este valor alcanza el $25 \%$ del valor de la meseta, puede inferirse que la variabilidad espacial se presenta a una escala menor de la mínima distancia considerada o a que existe un error experimental. En este caso es despreciable y evidencia un buen ajuste del semivariograma.

Finalmente, en la tercera y última fase del análisis, se construyó la representación gráfica bidimensional de la estimación kriging, lo que permitió identificar las manzanas con mayor índice de recipientes (figura 3 ).

\section{Discusión}

Con el uso de la estadística espacial se estudió la distribución en el espacio del indicador entomológico de $A$. aegypti correspondiente al índice de recipientes. Se empleó la función semivariograma y se construyó una representación gráfica del comportamiento de esta variable con la estimación geoestadística conocida como 


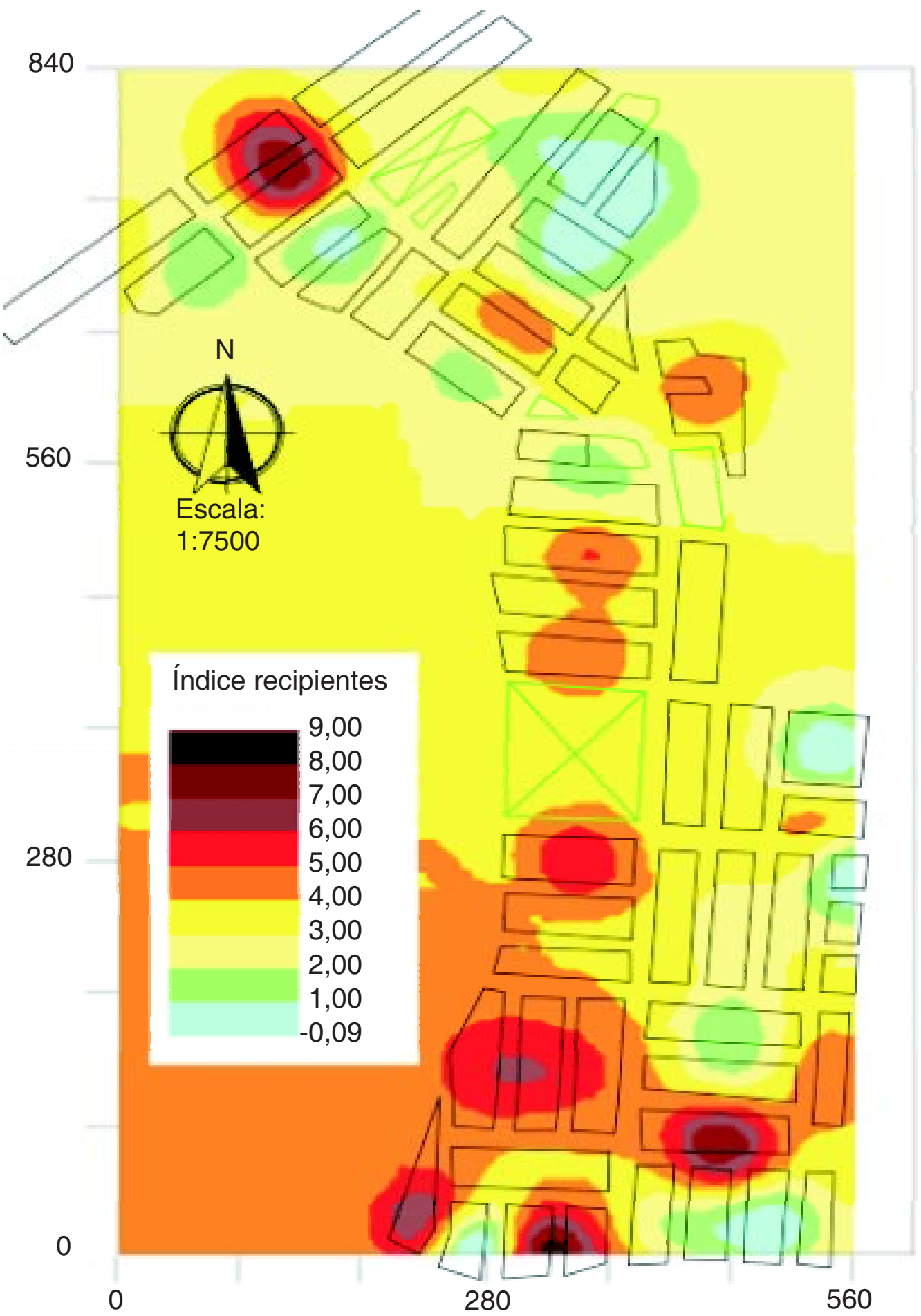

Figura 3. Representación gráfica bidimensional del kriging de la variable índice de recipientes. 
kriging, particularmente el ordinario en bloques. El semivariograma isotrópico evidenció un buen ajuste al modelo de Gauss por presentar el valor de la meseta similar al valor de la varianza de la población y una pepita cercana a cero. El rango o distancia máxima a la cual se correlacionan los datos se calculó en $57,15 \mathrm{~m}$, lo cual indica que los focos de infestación sólo inciden en las manzanas inmediatamente circundantes; su valor podría estar influido por la magnitud del tránsito de personas y vehículos, así como por la disponibilidad de alimento y sitios de cría, aduciendo que este mosquito puede desplazarse a mayores distancias.

El mapa de la distribución del índice de recipientes podría constituirse como una herramienta importante en la planificación de acciones en contra de $A$. aegypti, al indicar específicamente las áreas donde se hacen necesarias medidas de control más intensas de acuerdo con la presencia de focos de infestación, que busquen interrumpir o, al menos, minimizar el umbral de transmisión, en caso de brote o epidemia, aunque no se llegue a su completa erradicación (12). Asimismo, la implementación de medidas preventivas en zonas críticas en periodos específicos, requiere de este tipo de herramientas y métodos de vigilancia sensibles a la detección o predicción de incrementos súbitos en el crecimiento de la población del mosquito (13).

Además de determinar prioridades de acción, este tipo de estudios permiten identificar la población humana en riesgo y los factores favorables en el establecimiento de poblaciones densas de esta especie de mosquito (14).

En lo que respecta a la vigilancia entomológica, éste es un claro ejemplo de cómo acopiar, examinar, analizar e interpretar este tipo de información para así, no sólo adoptar, sino evaluar las medidas de intervención que se realicen en un lugar y momento determinados.

En definitiva, la prevención y el control de esta enfermedad requieren de nuevas tecnologías en cuanto a vigilancia, basadas en estudios de campo que incluyan la biología de poblaciones y la distribución espacial del vector en su ambiente natural. Los sistemas de información geográfica y la geoestadística posibilitan la obtención rápida de mapas donde se identifican los niveles de infestación del vector y su localización, lo que permite la orientación de las actividades de control del mosquito. Las características de estas técnicas de geoprocesamiento hacen de ellas herramientas poderosas en la planificación, la vigilancia y la evaluación de los programas de control. Muchos países han adoptado el análisis espacial de indicadores entomológicos dirigido a vigilar y controlar las poblaciones de vectores, principalmente en África, donde estas técnicas se orientan a mosquitos del género Anopheles, aunque los pocos estudios enfocados a $A$. aegypti han resultado promisorios en lo que respecta a vigilancia entomológica (13-15).

\section{Agradecimientos}

A la Secretaría Seccional de Salud del Meta, a Marina Stella González, Jorge Romero, Luz Stella Buitrago, Reinaldo Niño, Cateryn Romero, Ana María Gómez y Alexsy Ruiz; y a los auxiliares Maricela Porras, Lina Marcela Velásquez, Ruth Benavides, Armando Escobar, Mónica Mejía y Leidy Moreno.

\section{Conflicto de intereses}

El autor declara que no existe conflicto de intereses que pueda afectar los resultados de este trabajo.

\section{Financiación}

El presente trabajo fue financiado por la Gobernación del Meta, Secretaría Seccional de Salud.

\section{Referencias}

1. Schatzmayr H. Viroses emergentes e remergentes. Cad Saúde Publica. 2001;7(Suppl.):209-13.

2. Tinker M, Olano V. Ecología del Aedes aegypti en un pueblo de Colombia, Sur América. Biomédica. 1993;13:5-14.

3. Fernández W, lannacone J, Rodríguez E, Salazar N, Valderrama B, Morales A. Comportamiento poblacional de larvas de Aedes aegypti para estimar los casos de Dengue en Yurimaguas, Perú, 2000- 2004. Rev Peru Med Exp Salud Pública. 2005;22:175-82.

4. Taylor L. Assessing and interpreting the spatial distributions of insect populations. Annu Rev Entomol. 1984;29:321-57. 
5. Niño L. Características de las comunidades de Diptera (Arthropoda: Insecta) y su relación con el paisaje en la altillanura de la Orinoquia (Meta, Colombia) (trabajo de grado). Bogotá D.C.: Universidad Nacional de Colombia; 2005.

6. Liebhold AM, Rossi RE, Kemp WP. Geostatistics and geographic information systems in applied insect ecology. Annu Rev Entomol. 1993;38:303-27.

7. Burrough $\mathbf{P}$, MacDonell R. Principles of geographical information systems. New York: Oxford University Press; 1998.

8. Moral F. Aplicación de la geoestadística en las ciencias ambientales. Ecosistemas. 2004;13:78-86.

9. Isaaks E, Srivastava R. An introduction to applied geostatistics. New York: Oxford University Press; 1989.

10. Dale $\mathbf{M}$, Dixon $\mathbf{P}$, Fortin $\mathbf{M}$, Legendre $\mathbf{P}$, Myers $\mathbf{D}$, Rosemberg M. Conceptual and mathematical relationships among methods for spatial analysis. Ecography. 2002;25:558-77.
11. Journel A, Huijbregts C. Mining geostatistics. New York: Academic Press; 1978.

12. Kyle J, Harris E. Global spread and persistence of dengue. Annu Rev Microbiol. 2008;62:71-92.

13. Regis L, Monterior AM, Varial MA, Silveira JC Jr, Furtado AF, Acioli RV, et al. Developing new approaches for detecting and preventing Aedes aegypti population outbreaks: basis for surveillance, alert and control system. Mem Inst Oswaldo Cruz. 2008;103:50-9

14. Lagrotta M, Silva W, Souza-Santos R. Identification of key areas for Aedes aegypti control through geoprocessing in Nova Iguaçu, Rio de Janeiro State, Brazil. Cad. Saúde Pública. 2008;24:70-80.

15. Fantinatti E, Duque J, Silva A, Navarro-Silva M. Abundância e agregação de ovos de Aedes aegypti L. e Aedes albopictus (Skuse) (Diptera: Culicidae) no Norte e Noroeste do Paraná. Neotrop Entomol. 2007;36:960-5. 